\title{
Introduction to the Special Issue of Articles from the 2007 National Survey of Children's Health
}

\author{
Michael D. Kogan • Reem M. Ghandour • \\ Ashley H. Schempf
}

Published online: 6 April 2012

(C) Springer Science+Business Media, LLC (outside the USA) 2012

Children's health and well-being can be measured using a variety of metrics. Very often these measures are drawn from data systems which provide primarily national estimates of domain-specific indicators. The National Survey of Children's Health (NSCH) is unique among these systems in that it collects information on a comprehensive set of physical, mental and social health indicators among children, their access to and utilization of health services as well as family and community level factors that have been shown to influence health and development. The survey is designed to provide both national- and state-level estimates which are necessary for the development, implementation, and monitoring of effective health promotion and disease prevention programs for children and their families. As such, the NSCH fills a critical void and is the only data system that provides comparable estimates of children's health from birth to age 17 across every state in the nation. Reflecting a sustained commitment on the part of the Health Resources and Services Administration's Maternal and Child Health Bureau (MCHB), in collaboration with the Centers for Disease Control and Prevention's National Center for Health Statistics (NCHS), the $2007 \mathrm{NSCH}$ is the second such effort, building on the success of the first NSCH in 2003 to collect accurate and timely information on the health and wellbeing of children and their families with a third round of the

The views in this article are those of the authors and not necessarily those of the Health Resources and Services Administration of the US Department of Health and Human Services.

M. D. Kogan $(\square)$ - R. M. Ghandour · A. H. Schempf

U.S. Department of Health and Human Services, Health

Resources and Services Administration, Office of Epidemiology,

Policy and Evaluation, Maternal and Child Health Bureau,

Rockville, MD, USA

e-mail: mkogan@hrsa.gov
NSCH (2011) soon to be publicly available. The 2007 $\mathrm{NSCH}$ not only provides updated estimates of child health at both national and state levels but, coupled with the 2003 $\mathrm{NSCH}$, also provides the capacity to evaluate national, regional, and state trends as well as the impact of federal and state-level programs and policies.

The 2007 NSCH was fielded between April 2007 and July 2008 yielding 91,642 completed child-level interviews across the United States including 1,700-1,900 interviews in each State. Consistent with the original NSCH in 2003, the survey was conducted as a module of the State and Local Area Integrated Telephone Survey Program (SLAITS) which utilizes the sampling frame of the National Immunization Survey (NIS). The NIS is a random-digit dial telephone survey that uses computer-assisted telephone interview (CATI) technology to contact and interview households. In order to identify eligible subjects, households were screened for the presence of a child under the age of 18 years; if more than one child resided in the household, one was randomly selected to be the subject of the interview. Respondents were adults knowledgeable about the child's health. In nearly three-quarters of interviews this was the child's mother while $20.5 \%$ of the remaining interviews were conducted with the child's father, $4.2 \%$ were conducted with a grandparent and $1.8 \%$ with other relatives or guardians. Interviews were conducted in English, Spanish, Mandarin, Cantonese, Vietnamese, and Korean. Overall, $5.3 \%$ of interviews were conducted in Spanish and $0.2 \%$ were conducted in one of the four Asian languages [1]. The overall response rate was $51.2 \%$ and the interview completion rate was $66.0 \%$ for all households with children [2].

Similar to the 2003 survey, the 2007 NSCH contained "core" survey items in several domains, including: health and functional status, health care financing, access and 
utilization, family health and functioning, and neighborhood and community characteristics. Also included were agespecific modules for early childhood (0-5 years) and middle childhood/adolescence (6-17 years) which were designed to capture information on the presence of behavioral and social factors at critical periods that can impact the health and wellbeing of children into adulthood. The survey design also supported the collection of data on specific subgroups of children including those with special health care needs, and American Indian and Alaska Native and Asian children in selected states. Importantly, similarities between the 2003 and $2007 \mathrm{NSCH}$ allowed for the exploration of temporal patterns for a range of health indicators.

The 18 articles in this special issue featuring data from the 2007 NSCH highlight the breadth of topics covered in the survey and the unique design and capabilities with both national and state-level estimates. The articles address a wide range of topics, including breastfeeding, environmental tobacco smoke exposure, obesity, child development, behavioral problems, and autism, as well as access and utilization of health care including Human papilloma virus (HPV) vaccination, preventive dental visits, insurance, and the presence of a medical home. Some papers examined special populations and exposures like American Indian/ Alaska Native children, those with food allergies, and the impacts of residential mobility and Hurricane Katrina. The articles include national, regional, state-level and statespecific analyses, some of which employ sophisticated techniques like multilevel modeling, propensity scores, or innovative approaches that can be used to evaluate trends and policy impacts with both 2003 and 2007 survey data.

Eleven of the articles in this special issue utilized staterepresentative data to identify geographic disparities, explore the impact of state-level policies over time, and highlight particular state or regional challenges facing children and their families. Kramer and Dunlop [3] estimated the prevalence of HPV vaccination among adolescent girls in all 50 states. After adjustment for sociodemographic characteristics, they found significant state-level variation in HPV vaccine coverage among US girls aged 12-17 years which was largely explained by variability in reported clinician counseling. Geographic variation in clinical quality indicators was also investigated by Zickafoose et al. [4] who estimated the prevalence of medical home access by state of residence and insurance type. Through random-effects multilevel logistic regression, they found the prevalence of medical home access varied significantly across states for both publicly- and privately-insured children and that this variation was driven primarily by lower access among traditionally vulnerable groups rather than state-level policies or characteristics.

Similar to Zickafoose et al., several other papers used the NSCH in conjunction with other data sources to explore the potential of state-level policies to address factors known to adversely impact the long-term health and wellbeing of children. Belanoff et al. [5] examined whether state variability in race/ethnicity-specific breastfeeding practices and disparities could be explained by state sociodemographic composition or the availability of maternity care services related to breastfeeding. They concluded that while maternity care services were positively associated with breastfeeding overall, state-level differences in the availability of these services could not explain a substantial portion of breastfeeding variability. Bethell and colleagues [6] examined state-requirements of school-based case management for students with disabilities in relation to school success factors by special health care need (SHCN) status and emotional, behavioral, and developmental (EBD) problems. They found that SHCN, and in particular those of a behavioral or developmental nature, increased the risk for factors known to impede school success, but that the presence of a case management program lowered the odds of grade repetition among these high-risk students. The impact of state-level school-based policies were also investigated by Riis and colleagues [7] who studied the association between physical activity and nutrition policies and the prevalence of childhood obesity using 2 years of the NSCH. They found a positive association between state policies in these domains and increased childhood obesity but concluded that the concomitant rise in both indicators was reflective of the adoption of policies by states with increasing levels of childhood obesity rather than a causal link between policy implementation and obesity. Similarly, Hawkins et al. [8] linked 2 years of NSCH data with state data on tobacco-related legislative and tax policies to examine their impact on tobacco use in households with children and disparities in secondhand smoke exposure using both causal inference techniques, known as differences-in-differences models, and cross-sectional models for comparison. They found that every $\$ 1.00$ increase in cigarette excise tax between 2001 and 2005 was associated with a 4 percentage-point decrease in household tobacco use between survey years while conventional cross-sectional models produced biased results.

Two papers used state-level data to explore distinct, yet related, aspects of insuring children living in poverty. Crocetti and colleagues [9] identified state-, child-, and family-level characteristics associated with uninsurance among children eligible for publicly-funded coverage while Colby et al. [10] explored insurance adequacy and health care access among publicly-insured children within the context of state Medicaid expenditures. Crocetti et al. found that approximately 3.2 million U.S.-born children were eligible for health coverage under Medicaid or CHIP but were not insured. Although child-level factors such as age were associated with uninsurance, significant state- 
level variation was found with nearly half of eligible-butuninsured children residing in one of five states. Using a measure of per-member-per-month Medicaid expenditures, Lipson and colleagues found that publicly-insured children living in states with above average per-child expenditure had significantly increased odds of having insurance that adequately covered their needs, particularly those for mental health services. The authors also identified highvalue states with lower-than-median spending and higherthan-median adequacy and access.

Three other submissions utilized state-level data to explore challenges specific to a particular state, region, or population. In response to the Georgia Title V Needs Assessment completed in 2010, Ogbuanu and colleagues [11] investigated factors associated with access to and quality of care for Georgian children by merging data from the $2007 \mathrm{NSCH}$ with county-level data from the Area Resource File. While the majority of Georgian children were found to have access to health care, reported quality differed by continuity and adequacy of insurance coverage suggesting the need to identify new and innovative methods of extending and retaining comprehensive coverage for children in Georgia. In contrast, Stehling-Ariza and colleagues [12] focused on a single measure of health care access-a personal health provider-in evaluating the impact of exposure to Hurricane Katrina. Using propensity scores, they matched children exposed to Katrina from the 2007 Gulf Coast Child and Family Health Study to otherwise similar children residing in unaffected but demographically similar gulf states from the NSCH. Their analysis showed that exposed children were $20 \%$ less likely to have a personal healthcare provider compared to unexposed children. Results from traditional regression analyses were compared and the use of propensity score methods was discussed. The article by Barradas et al. [13] focused on evaluating medical home access among American Indian and Alaska Native children (AIAN) using data from 7 states that could report this race category. The authors found that less than one-half of AIAN children had access to a medical home compared to $58.3 \%$ of nonHispanic White children in the same states. The study highlights areas of particular concern including the provision of care coordination and family-centered care, as well as the potential for differential medical home access among users of the Indian Health Service by insurance type.

Among the remaining national-level analyses, several focused on specific groups of developmental activities, problems, or disorders. Kenney [14] examined factors associated with interactive play between young children and their peers and parents that may enhance early learning and socialization. Results indicate that while various sociodemographic and parental factors broadly influenced young children's experience of being read or sung to, participating in peer play, and being taken on family outings, neighborhood factors such as amenities and safety were more strongly associated with peer play and family outings. Singh and Ghandour [15] also explored the impact of neighborhood social conditions and household SES on the prevalence of behavioral problems among school-aged children. Using both a factor analytic index and dichotomous measure of serious behavioral problems (SBP), the authors found that both neighborhood social disadvantage and lower household SES were strongly associated with behavioral problems; children from low-SES households and disadvantaged neighborhoods had 1.9-3.7 times higher odds of SBPs than children from the most advantaged households even after controlling for neighborhood conditions. Finally, the article by Schieve and colleagues [16] similarly focused on a single family of conditions: autism spectrum disorders (ASD) but used both years of the NSCH to explore the observed twofold increase in the prevalence of ASD. By looking at changes between the 2003 and 2007 survey within 1-year birth cohorts who had aged 4 years between surveys, they were able to hold genetic and non-genetic prenatal factors constant and better isolate an impact of increased diagnosis. In addition to measurement differences, the authors concluded that increased population awareness and access to ASD diagnostic services contributed substantially to the increase in prevalence and that their impact on ASD prevalence estimates is likely to persist.

Among the studies that examined multiple physical, emotional, and behavioral developmental indicators, two evaluated the effects of specific exposures (low birthweight and residential mobility) while another developed a comprehensive index of child well-being. Salas, Xaverius and Chang [17] examined the impact of low birthweight (LBW) on physical and developmental conditions among children aged 5 years and younger and whether medical home access mitigated the adverse developmental effects of LBW. Their results show that LBW children were almost twice as likely as those born at a normal weight to have a physical or developmental condition and poorer overall health, irrespective of medical home access-a finding that suggested universal benefit of receiving care within a medical home regardless of birthweight. Busacker and Kasehagen [18] used multivariable analyses to explore the association of residential mobility with measures of child health and wellbeing. Their results show that children who moved $\geq 3$ times were more likely to have poorer overall physical and oral health, to have $\geq 1$ moderate/severe chronic conditions, to be uninsured or have had periods of no coverage in the prior year, and to lack a medical home compared to children with no reported residential moves. Utilizing the full complement of child health indicators, Moore and colleagues [19] describe the development and potential applications for a multifaceted index of child well-being comprised of four 
developmental domains: physical health, psychological health, social health, and educational achievement and cognitive development. The authors emphasize the importance of focusing on positive indicators as opposed to negative measures traditionally favored, as well as measures of overall well-being rather than narrow indicators of specific aspects of child health.

The supplement also features two articles that examined different aspects of health services utilization. In an article on access to and use of preventive oral health care, Bell and colleagues [20] estimated associations between perceived oral health status and receipt of a preventive dental health visit in the year prior to the survey. In addition to disparities in oral health status, their results indicate that receipt of preventive oral health care varies differentially by age and need. The authors emphasize that public health efforts should focus on promoting early and ongoing preventive oral health care, rather than care in response to identified problems. By contrast, Branum and colleagues [21] examined a variety of health care utilization measures among a specific population of children who have food allergies. The authors explored differences by food allergy severity and sociodemographic characteristics. Children with moderate/severe allergies were more likely than those with mild allergies to use an Individual Education Plan, have seen a specialist for care in the past year, and require more care than usual. The authors found no disparities in health care use by race/ethnicity or income.

Taken together, the articles featured in this special issue are a testament to the breadth and depth of information available in the $2007 \mathrm{NSCH}$. As illustrated in this collection of manuscripts, the analytic potential for the data alone and in conjunction with other systems is substantial and holds the potential to address a multitude of research questions at both state and national levels. We hope these data, in concert with those from previous and future editions of the Survey, will be used by researchers, policy makers, advocates, students, and consumers to improve the health and well-being of children and families across the nation. Data are available for download from the National Center for Health Statistics at: http://www.cdc.gov/nchs/slaits/nsch.htm. Users can also search the data interactively through the MCHB-funded Data Resource Center at: http://childhealthdata.org/home. Finally, a series of Chartbooks on children's health topics from the $2007 \mathrm{NSCH}$ are available electronically at: http://mchb. hrsa.gov/.

\section{References}

1. Maternal and Child Health Bureau. (2009). The Health and WellBeing of Children: A Portrait of the States and the Nation 2007.
Rockville, MD: Health Resources and Services Administration, US Department of Health and Human Services.

2. Blumberg, S. J. F. E., Frasier, A. M., Satorius, J., Skalland, B. J., Nysse-Carris, K. L., Morrison, H. M., Chowdhury, S. R., \& O'Connor, K. S. (2009). Design and Operation of the National Survey of Children's Health, 2007. Hyattsville, Maryland: US Department of Health and Human Services, Centers for Disease Control and Prevention, National Center for Health Statistics.

3. Kramer, M. R., \& Dunlop, A. L. (2011). Inter-state variation in human papilloma virus vaccine coverage among adolescent girls in the 50 US States, 2007. Maternal and Child Health Journal. doi:10.1007/s10995-012-0999-6.

4. Zickafoose, J. S., Gebremariam, A., \& Davis, M. M. (2011). Medical home disparities for children by insurance type and state of residence. Maternal and Child Health Journal. doi: 10.1007/s10995-012-1008-9.

5. Belanoff, C., McManus, B., Carle, A. C., McCormick, M. C., \& Subramanian, S. V. (2011). Racial/ethnic variation in breastfeeding across the US: A multilevel analysis from the National Survey of Children's Health, 2007. Maternal and Child Health Journal. doi:10.1007/s10995-012-0991-1.

6. Bethell, C., Forrest, C. B., Stumbo, S., Gombojav, N., Carle, A. C., \& Irwin, C. E. (2011). Factors promoting or potentially impeding school success: Disparities and state variations for children with special health care needs. Maternal and Child Health Journal. doi:10.1007/s10995-012-0993-z.

7. Riis, J., Grason, H., Strobino, D., Ahmed, S., \& Minkovitz, C. (2011). State school policies and youth obesity. Maternal and Child Health Journal. doi:10.1007/s10995-012-1000-4.

8. Hawkins, S. S., Chandra, A., \& Berkman, L. (2011). The impact of tobacco control policies on disparities in children's secondhand smoke exposure: a comparison of methods. Maternal and Child Health Journal. doi:10.1007/s10995-012-0996-9.

9. Crocetti, M., Ghazarian, S., Myles, D., Ogbuoji, O., \& Cheng, T. L. (2011). Characteristics of children eligible for public health insurance but uninsured: Data from the 2007 National Survey of Children's Health. Maternal and Child Health Journal. doi: 10.1007/s10995-012-0995-x.

10. Colby, M. S., Lipson, D. J., \& Turchin, S. R. (2011). Value for the money spent? Exploring the relationship between expenditures, insurance adequacy, and access to care for publicly insured children. Maternal and Child Health Journal. doi:10.1007/ s10995-012-0994-y.

11. Ogbuanu, C., Goodman, D., Kahn, K., et al. (2011). Factors associated with parent report of access to care and the quality of care received by children 4 to 17 years of age in Georgia. Maternal and Child Health Journal. doi:10.1007/s10995-012-1002-2.

12. Stehling-Ariza, T., Park, Y. S., Sury, J. J., \& Abramson, D. (2011). Measuring the impact of Hurricane Katrina on access to a personal healthcare provider: The use of the National Survey of Children's Health for an external comparison group. Maternal and Child Health Journal. doi:10.1007/s10995-012-1006-y.

13. Barradas, D. T., Kroelinger, C. D., \& Kogan, M. D. (2011). Medical home access among American Indian and Alaska native children in 7 states: National Survey of Children's Health. Maternal and Child Health Journal. doi:10.1007/s10995-012-0990-2.

14. Kenney, M. K. (2011). Child, family, and neighborhood associations with parent and peer interactive play during early childhood. Maternal and Child Health Journal. doi:10.1007/ s10995-012-0998-7.

15. Singh, G. K., \& Ghandour, R. M. (2011). Impact of neighborhood social conditions and household socioeconomic status on behavioral problems among US children. Maternal and Child Health Journal. doi:10.1007/s10995-012-1005-z.

16. Schieve, L. A., Rice, C., Yeargin-Allsopp, M., et al. (2011) Parentreported prevalence of autism spectrum disorders in US-born 
children: An assessment of changes within birth cohorts from the 2003 to the 2007 National Survey of Children's Health. Maternal and Child Health Journal. doi:10.1007/s10995-012-1004-0.

17. Salas, J., Xaverius, P. K., \& Chang, J. J. (2011). Does a medical home influence the effect of low birthweight on health outcomes? Maternal and Child Health Journal. doi:10.1007/s10995-012-1003-1.

18. Busacker, A., \& Kasehagen, L. (2011). Association of residential mobility with child health: An analysis of the 2007 National Survey of Children's Health. Maternal and Child Health Journal. doi:10.1007/s10995-012-0997-8.

19. Moore, K. A., Murphey, D., \& Bandy, T. (2011). Positive child well-being: An index based on data for individual children.
Maternal and Child Health Journal. doi:10.1007/s10995-0121001-3.

20. Bell, J. F., Huebner, C. E., \& Reed, S. C. (2011). Oral health need and access to dental services: Evidence from the National Survey of Children's Health, 2007. Maternal and Child Health Journal. doi:10.1007/s10995-012-0992-0.

21. Branum, A. M., Simon, A. E., \& Lukacs, S. L. (2011) Among children with food allergy, do sociodemographic factors and healthcare use differ by severity? Maternal and Child Health Journal. doi:10.1007/s10995-012-1009-8. 